\title{
Políticas y programas de Estado para la salud rural en América Latina: una revisión de la literatura*
}

\section{State policies and \\ programs for rural health}

\author{
in Latin America: a review \\ of the literature
}

Lorena Parada Parra**

Recibido: 23 de Octubre de 2018

Revisado: 30 de Noviembre de 2018

Aprobado: 5 de diciembre de 2018

\section{Resumen}

El objetivo de este trabajo es identificar las principales políticas y programas estatales en Latinoamérica para el fomento de la salud rural. Se utiliza como método el estudio transversal retrospectivo para determinar las principales leyes que han permitido el

* Artículo producto de revisión de literatura realizada para la línea de Profundización en Gestión de las Organizaciones, Planes y Programas de Salud Pública, hace parte del proyecto sobre políticas públicas en Salud. Cómo citar este artículo: Montalvo, Y. A. y Jácome, V. J. (2019). Elementos para la construcción de un modelo de gestión dirigido a la banca comunal en Ecuador. Revista CIFE: Lecturas de Economía Social, 20(33), 79-94. DOI: https://doi. org/10.15332/22484914.4878

** Enfermera de la Universidad Nacional de Colombia. Fundación Cardio infantil. Estudios de Maestría en Salud Pública. 
surgimiento de políticas y programas rurales para la promoción de la salud, dirigidos a pobladores de zonas rurales en Latinoamérica, con ello se comprenden las acciones que se han trabajado en el ámbito rural. La revisión de la literatura muestra que existen diversos programas que dan respuesta parcial a los problemas de salud existentes en las zonas rurales. Respecto a los programas de fomento rural en América Latina, la mayor parte son programas de lucha contra la pobreza, algunos de ellos tienen impacto en los indicadores sanitarios, sin embargo, existen programas que ya expiraron y para el caso de Colombia, es necesario tener más en cuenta las zonas rurales para dar mayor alcance en salud.

Palabras clave: salud rural, desarrollo rural (fuente DeCs, MeSH).

Clasificación JEL: I18, I38, R58

\section{Abstract}

The object of this work is to identify the main state policies and programs in Latin America for the promotion of rural health. The retrospective cross-sectional study is used as method to determine the main laws that have allowed the emergence of rural policies and programs for the promotion of health, aimed at rural inhabitants in Latin America, thereby comprising the actions that have been performed in the rural area. The review of the literature shows that there are several programs that partially respond to the health problems existing in rural areas. Regarding rural development programs in Latin America, most of them are anti-poverty programs, some of them have an impact on health indicators, however, there are programs that have already expired and for the case of Colombia, it is necessary to consider rural areas more to give greater scope in health.

Keywords: rural health, community health, rural development (Source DeCs, MeSH).

Classification JEL: I18, I38, R58 


\section{Introducción}

Ante la crisis económica de la mayor parte de los países latinoamericanos en las décadas de los 70 hasta principios de los 90, cada Estado ha trabajado en la introducción de políticas de tipo estructural cuyo desenlace ha impactado al mercado en su privatización y su explotación a través de la apertura económica (Rodríguez, 2011), de aquí surgen políticas que dejan de lado a un sistema de intervención, creando vacíos en las instituciones públicas rurales, agudizando a su vez condiciones de desigualdad (Brumer, 2003); por otra parte, se evidencia el abandono a los pequeños productores, ya que los gremios industriales tienen mayor atención del Estado por su contribución económica y beneficios secundarios a su actividad (Machado, 2010). Las políticas rurales han quedado reducidas a programas para la lucha contra la pobreza, como se identifica en la gestión de diversos países de Latinoamérica (Zurbriggen y Travieso, 2016), sin embargo, se evidencia mayor preocupación por el impacto económico de la pobreza rural que por la salud de estos.

Existen diferentes dinámicas demográficas en Latinoamérica que buscan repensar la salud en términos de necesidades latentes de acuerdo al grupo poblacional más dependiente de servicios de salud, así como se encuentra un significativo aumento entre la población mayor de edad con una marcada disminución en la natalidad (Felitti, 2009). En los países latinoamericanos la población mayor de 60 años continúa en aumento, países como Argentina, Cuba y Uruguay en la actualidad se encuentran en un periodo de envejecimiento avanzado, con al menos el $10 \%$ de población adulto mayor y con proyección de triplicarse entre los años 2000 y 2050 (Arriagada, Aranda y Divisi, 2005).

Existen aún dificultades en términos de equidad, prestación de servicios de salud, incluso sistemas de salud organizados para la población rural en diferentes países de Latinoamérica (Pérez y Arrivillaga, 2017); en términos de cobertura y acceso aún hay mucho por discutir, dado el limitado acceso hay en países como Chile, Bolivia, Guatemala, Colombia, Paraguay, Panamá, República Dominicana y Perú, así mismo, y de manera particular, hay registros sobre la insatisfacción en los servicios ofrecidos al sector rural en países como el Salvador (Acosta, Sáenz, Gutiérrez y Bermúdez, 2011; BBC News Mundo, 2017).

\section{Antecedentes de políticas y programas para el fomento de la salud rural en Latinoamérica}

Existe una estrecha relación entre la pobreza y su resultado en las condiciones de salud de una población (Ortiz, Pérez y Tamez, 2014), mostrando así que los mayores índices de mortalidad corresponden a los sectores más pobres, y suelen ser más productivos aquellos que gozan de buena salud en términos económicos; alrededor del 60 \% de la 
población rural vive en condiciones de pobreza o indigencia (Organización Mundial de la Salud, 2003). Las poblaciones más vulnerables suelen encontrarse en zonas dispersas, donde los servicios de salud difícilmente pueden llegar, evidenciándose como desamparo hacia la población rural por parte de los estados latinoamericanos (Salgado, González, Jaurégui y Bonilla, 2005), para demostrar este argumento se hace una revisión de las diferentes estrategias gubernamentales para combatir la pobreza rural y de esa manera identificar estrategias para el fomento de la salud rural.

\section{Políticas rurales en Latinoamérica}

Entiéndase por política como una actividad consistente en la toma de decisiones para actuar frente a determinados aspectos que requieren atención por parte de entidades para la generación de planes, programas y proyectos, en este caso para el fomento de la salud rural (Canel, 2006); una característica de las políticas es que son flexibles y permiten cambios de acuerdo con las dinámicas poblacionales. A partir de la revisión se relacionan algunas de las políticas para Latinoamérica.

Para el año de 1963, Brasil establece como objetivo la protección a los trabajadores rurales, brindando recursos para la eliminación de desigualdades entre la concepción de lo urbano y lo rural por medio del Fondo de Asistencia y Previsión del Trabajador Rural (FUNFURAL), con ello surgieron una serie de modificaciones en la Constitución política, las cuales fueron efectivas en 1991, y se dio paso a la creación de un sistema de previsión social que cubría a trabajadores formales e informales del sector rural. FUNRURAL ha tenido un impacto positivo para el bienestar de la población más pobre del sector rural, y los aportes del sistema de previsión equivalen a una fracción de la renta domiciliaria rural, sin embargo, no ha modificado la estructura de producción del sistema económico (Breu, Guggenbichler y Wollmann, 2003). Aunque FUNFURAL ha tenido algunas variaciones de funcionamiento, sigue acompañando a la población rural de Brasil.

Recientemente en Chile surge la Política de desarrollo rural (2014-2024), ante un diagnóstico realizado por la Organización para la Cooperación y el Desarrollo Económicos (OCDE) donde se había determinado la falta de una política rural por lo que surge una propuesta donde se reconoce la ruralidad como algo esencial que debe ser protegido por el estado, dirigido hacia un desarrollo sustentable, trabajando por mejorar la calidad de vida para la población rural, con ello apuntan a 5 ejes estratégicos que cubren: bienestar social de la población rural, oportunidades económicas en territorio rural, protección de la cultura rural y gobernabilidad de la política de desarrollo rural; con ello se plantea enfrentar los nuevos desafíos de orden social, económico y ambiental (Comité Técnico Interministerial, 2014). 
En el campo de lo rural, y no ajeno al constructo de otros países, Colombia apunta hacia la política pública en pro de la mujer rural implementada a partir del decreto 2145 de 2017, por medio del cual se realiza un seguimiento y evaluación de los programas y leyes en favor de la mujer rural, cuyo principal interés es contribuir en la disminución de las brechas de género para favorecer la autonomía de las mujeres, a la vez eliminar dificultades de acceso a las oportunidades que provean diferentes instituciones a su favor, para ello se fundó el comité interinstitucional de seguimiento para cumplimiento de los objetivos propuestos (Decreto 2145, 2017). El equipo de trabajo cuenta con un comité para la dirección de la mujer rural y una consejería presidencial para la equidad de la mujer (Ministerio de Agricultura, 2018).

Por otra parte, en Nicaragua se crea el Instituto de Desarrollo Rural (IDR), con el fin de fortalecer a pequeños y medianos productores, para impulsar el comercio en la región, con ello se pretende mejorar el ingreso a los servicios financieros de miles de familias rurales, con el apoyo en el mejoramiento de las vías de transporte para facilitar el comercio y la inclusión de los campesinos pobres para la ejecución de proyectos agroindustriales; a partir de las necesidades identificadas en términos de desarrollo rural, inicia del año 2000 a 2001 la implementación de un posgrado en desarrollo rural en grado de maestría, con el fin de fortalecer el capital humano para el desarrollo de planes y políticas en el sector rural (Romero, Ferrero, Pérez, Baselga y Rodríguez, 2000).

La creación de diversas políticas relacionadas con condiciones de pobreza identificadas en la región también ha sido propósito para Nicaragua por medio del Fondo Social Suplementario, establecido a partir del decreto 46-98 de 1998 (Soto, 2000), dados los altos índices de pobreza en la población con bajos indicadores de salud y educación en la población rural, su finalidad fue el financiamiento de programas de índole social por tres años (República de Nicaragua, 1998). En el país se trabajó en un plan para aumentar la cobertura en la prestación de servicios de salud para los habitantes más pobres de las zonas rurales, y el fondo tuvo un papel fundamental en su desarrollo. El trabajo a partir del Fondo Social Suplementario dio origen al programa de inmunizaciones, vigilancia y control de cáncer cérvico uterino, malaria, dengue, dispensación gratuita de medicamentos y unido a ello el aseguramiento de personal de enfermería hacia las zonas rurales.

Para el desarrollo de la propuesta se trazaron objetivos como la reducción de la morbimortalidad en niños menores de 5 años a través del programa Integral para niños y la Atención de Enfermedades Prevalentes en la Infancia, por otra parte, se impulsaron brigadas de salud móviles que se han trabajado hace más de diez años para zonas de difícil acceso. El gobierno de Nicaragua diseñó además un modelo de salud familiar y comunitaria, el cual requirió capacitación del personal de salud para la atención en diferentes niveles. Instituciones como MIFAMILIA, Instituto Nicaragüense de Seguridad Social (INSS), cooperación internacional y el Ejército Nacional han sido participes activos de 
movilización social para llegar a población en extrema pobreza en zonas rurales con difícil acceso (Organización Panamericana de la Salud, 2008).

\section{Programas rurales en Latinoamérica}

Entiéndase un programa como el producto de una planeación, cuyo contenido encierra una serie de actividades para el alcance de los objetivos establecidos por una organización, parte inicialmente de una política. A través de los programas rurales se han establecido acciones para fomentar la salud rural.

Promover la igualdad para la población rural es uno de los objetivos de Chile, donde se ha contribuido por medio del Instituto Nacional de Desarrollo Agropecuario (INDAP), cuyo objetivo es el impulso del desarrollo económico sostenible, dando valor a la agricultura familiar campesina por medio del refuerzo del capital humano y productivo desde 1962, con ello se promueve el trabajo de pequeños agrícolas y campesinos a partir del desarrollo de tecnologías, economía y sociedad, a fin de elevar su capacidad productora. Lo más importante para el INDAP es el impulso de programas para el financiamiento de las familias campesinas, promoción de productos tradicionales, fortalecimiento de la agricultura familiar campesina e impulso de alianzas entre productores y actores públicos y privados para la canalización de recursos en favor de la ruralidad (Ministerio de Agricultura, 2016).

En Perú se crea para el año de 1981 el Programa Nacional de Manejo de Cuencas Hidrográficas y Conservación de Suelos (PRONAMACHCS), perteneciente al Ministerio de Agricultura, su principal objetivo es la lucha contra la pobreza rural; Perú cuenta con un vasto territorio rico en recursos naturales y por ellos lucha PRONAMACHCS, programa que representa un rubro importante del Ministerio y que tiene en cuenta a los campesinos de la Sierra con su rol fundamental en la conservación, por lo que por medio de capacitaciones impartidas por profesionales se infunden conocimientos técnicos hacia los habitantes para potenciar el manejo de los recursos naturales de manera sostenible (Lara, Pardo y Escalante, 2005).

En Perú, siguiendo la línea de trabajo hacia los hogares rurales, en 1991 se crea el Fondo de Compensación y Desarrollo Social (FONCODES), programa del Ministerio de Desarrollo e Inclusión Social (MIDIS), quienes trabajan a favor de familias campesinas con pobreza extrema en la difusión de oportunidades económicas que permitan su sustento. La propuesta de Perú toma en cuenta el desarrollo de capacidades productivas en las familias y su emprendimiento para la inserción competitiva en el mercado, a su vez fomenta la participación comunitaria para mejorar la inversión pública hacia los hogares en situación de pobreza extrema. En el año 2017 el Fondo recibió un 
reconocimiento en buenas prácticas de gestión pública (Fondo de Cooperación para el Desarrollo Social, 2017).

Para el caso de Colombia, existen antecedentes de programas como el Instituto Colombiano de Reforma Agraria INCORA, que existió hacia el año de 1961 e inicialmente tenía como fin trabajar contra el desplazamiento campesino por dificultad en la oportunidad de trabajo en las zonas rurales y carencia de tierras, así que lideró el programa de reforma agraria mediante adquisición y redistribución de tierras, por otra parte se crea el Sistema Nacional de Reforma Agraria y Desarrollo Rural a partir de la Ley 160 de 1994, la cual trabajaba los procesos relacionados con la redistribución de la tierra y economía campesina con mayor regulación a partir de reglamentación del Ministerio de Agricultura, lo cual generó dificultades administrativas para los campesinos (Gómez, 2016).

Colombia hacia la década de 1990 se vio enmarcada por un proceso de apertura económica como respuesta a las necesidades de la economía mundial, varios procesos se privatizaron a la vez que intervinieron procesos tecnológicos con miras a la acumulación de capital, sin embargo dichos procesos no fueron de la mano con el desarrollo rural, por lo que los campesinos en gran parte se vieron desamparados, sobre todo los pequeños productores, quienes fueron poco a poco quedando excluidos del proceso económico nacional, con ello surgieron condiciones de pobreza que no tardaron en incidir en procesos de salud y enfermedad, ello constituye en dinámicas de desigualdad, aún más marcados si se ven las diferencias entre el trabajador rural y urbano (Rodríguez y Benavidez, 2016).

Para el año de 1994, en Colombia nace la Red de Solidaridad Social como una estrategia de desarrollo social y humano, cuyo fin era llegar a la población más vulnerable en condición de pobreza, que por causa de vivir como población dispersa no es alcanzable por los diversos programas gubernamentales, por lo que quedan excluidos de beneficios de crecimiento económico; a partir de la Red de Solidaridad se buscaba mejorar la calidad de vida de la población más vulnerable por medio de la potenciación de las capacidades de participación comunitaria (Jaramillo, 2005).

Para Colombia, otro de los ejemplos de construcción para el desarrollo rural es el Programa de Desarrollo Rural con Equidad (DRE), antes llamado Agro Ingreso Seguro (AIS), creado por la Ley 1133 de 2007, destinado a la protección de los ingresos de los productores que se afecten por alteraciones secundarias al mercado externo, adicional pretende mejorar la competitividad en el sector agropecuario a nivel nacional, a través de créditos e incentivos a la productividad (Ley 1133, 2007). En la actualidad, Colombia no cuenta con una estrategia planificada para el desarrollo campesino ya que sus recursos se han distribuido para apoyar a empresarios agrícolas, lo que ha impactado en la 
persistencia de inequidad con relación a la población rural (Machado, 2010), siendo la pobreza, la desigualdad y el desempleo origen del desplazamiento rural (Acosta, 2013).

Por otra parte, Bolivia ha trabajado más los programas encaminados al sector salud para la ruralidad, por medio del Programa Mi Salud, se trata de una estrategia de atención primaria en salud, mediante la implementación de la política para la promoción y atención integral intercultural con participación comunitaria y control social SAFCI (Pérez y Arrivillaga, 2017), la cual busca que la comunidad deje la exagerada dependencia hacia el personal de salud y se haga cargo de su protección. La atención en salud con participación comunitaria es mucho más costo-efectiva y representa el modelo de atención primaria en salud (APS).

Para el desarrollo del proyecto fue necesaria la incorporación de médicos con funciones destinadas al primer nivel de atención para las zonas más apartadas del país, y con ello garantizar que la población acceda a la salud de manera gratuita y con calidad, dicho programa inició en el año 2013 (Ministerio de Salud Bolivia, 2015).

\section{Programas de transferencia monetaria condicionada en Latinoamérica}

Los programas de transferencia monetaria condicionada plantean la entrega de dinero a los beneficiarios quienes generalmente son población vulnerable, de acuerdo con el cumplimiento de requisitos que para efectos del contexto latinoamericano se direcciona a mejorar aspectos en salud (Valencia, 2008).

Diversos estudios muestran que los modelos de desarrollo rural están construidos con enfoques desiguales, de tipo económico (Kliksberg, 2002), sin negar el impacto que tienen las ayudas económicas al mejoramiento de la salud en la población rural, sirviendo como apalancamiento para salir de condiciones de pobreza extrema (Reyes, 2014); considerando la relevancia de la economía para los sectores productivos, se evidencia una falla en la integración del sector rural en las diferentes políticas de salud, configurados así como modelos excluyentes, ejemplo de ello están países como El salvador, Honduras, Guatemala y Nicaragua; sin embargo, existen excepciones, países como Brasil presentan una cobertura en salud del $92 \%$ en zonas rurales y en Chile la cobertura en salud solo es mayor 1,5 veces en el área urbana respecto de la rural, para la generalidad de los países solo un habitante rural de cada cinco recibe pensión o jubilación (Tokman, 2006).

Diferentes estados han coincidido en la creación de programas de transferencia como respuesta al déficit económico que presenta la población rural con su impacto secundario 
en calidad de vida y salud, como ejemplo de lo que se ha implementado a nivel gubernamental para las zonas rurales se muestran acciones en Argentina, con la Asignación Universal por Hijo (AUH) para la protección social en Argentina creado en 2009; para 2011 se adiciona la Asignación Universal por Embarazo (AUE), lo que significó una etapa nueva para la política social de Argentina, con ello se inició un proceso de inclusión entre desempleados y trabajadores informales, frecuentemente excluidos de las políticas. Con las asignaciones realizadas a los hogares se impactan los niveles de pobreza e indigencia (Pautassi, Arcidiácono y Straschnoy, 2013).

Por parte de Brasil, se creó en 2003 el programa Bolsa Familia, se trata de un programa de transferencia monetaria condicionada cuyo objetivo es el apoyo de las familias en situación de pobreza, ampliando a su vez el acceso a la educación y la salud. El programa tiene un alcance nacional, pese a ello tiene bajo costo para la nación, representando solo el 0.45 \% del PIB brasileño (División de Protección Social y Salud, 2016); otra de las estrategias para Brasil surge con la creación de LA Bolsa Verde, programa de transferencia de ingreso creado en 2011 en el marco del programa Brasil sin pobreza, dirigido a familias con condiciones de pobreza extrema que viven en áreas de conservación del medio ambiente, el fin del incentivo que brinda el programa es promover la conservación de los recursos para lograr una convivencia más sustentable (Ministerio de Medio Ambiente, 2011).

En Colombia, por otra parte para el año 2000 surge Más Familias en Acción, programa de transferencia monetaria condicionada, cuyo fin es brindar beneficios en asistencia escolar y salud a familias pobres, desplazadas víctimas del conflicto armado e integrantes de comunidades indígenas menores de 18 años, los beneficiarios pueden acceder de acuerdo a la clasificación del SISBEN (Medellín y Sánchez, 2015).

Para el caso de El Salvador, se crea en 2009 el programa Comunidades Solidarias Rurales, el cual busca promover la participación de la comunidad con énfasis en niñas, adolescentes y mujeres adultas para disminuir las brechas de género, adicionalmente tiene en cuenta a personas en situación de discapacidad. Su principal objetivo es brindar atención integral a familias excluidas socialmente o en condición de pobreza extrema, además de promover condiciones de igualdad en oportunidades entre comunidades (Salvador, 2012).

La mayor parte de transferencias se brindan por medio de bonos, como lo demuestra Ecuador con el Bono de Desarrollo Humano, creado en 2003, que reemplazó el programa de bono solidario que había iniciado funciones en 1998; este programa va dirigido a familias en condición de pobreza extrema, teniendo prelación en población de adultos mayores y discapacitados. Apoyados en el índice de bienestar para la identificación de los beneficiarios se les determina de acuerdo a la información socioeconómica de los hogares (Martínez, Borja, Medellín y Nadin, 2017). 
Por parte de México, se tiene el programa de Desarrollo Humano Oportunidades, el cual brinda transferencias a hogares pobres con la condición de promover inversión en capital humano de los hogares beneficiados; el programa promueve la asistencia escolar, adicionalmente entre las condiciones del beneficio requiere el cumplimento de condiciones de salud de acuerdo a las estrategias vivir mejor y el apoyo infantil vivir mejor (Hernández y Escobar, 2012); una particularidad con el programa de México es que más allá de la transferencia económica, el Gobierno busca promover y garantizar el acceso a derechos sociales de la población más vulnerable.

En Panamá existe la Red de Oportunidades Empresariales para Familias Pobres para la reducción de los niveles de pobreza con especial énfasis en las zonas rurales e indígenas, incentivando emprendimiento sostenible en sectores agropecuarios y turismo (República de Panamá, 2009).

Otro es el caso de Paraguay, con la creación de Tekoporá en 2005, su objetivo es la atención a familias con condición de pobreza extrema por medio de transferencias monetarias, el objetivo del programa es mejorar la calidad de vida de las personas a través del derecho al acceso a alimentación, salud y educación (CEPAL, 2005). Siguiendo la línea de educación y salud se encuentra el programa Juntos del Estado de Perú, surge por parte del Ministerio de Desarrollo e Inclusión Social y su misión es la promoción al acceso hacia los servicios de salud y educación de hogares en condición de pobreza, el incentivo se brinda siempre que las familias lleven a los niños y niñas a los centros de salud, colegio y controles prenatales en caso de gestantes (Sánchez, Melendez y Behrman, 2016).

En República Dominicana con la estrategia Progresando con Solidaridad creada en 2012, se plantea la reducción de los índices de pobreza mediante el fortalecimiento de la red de protección social, trabajando en inclusión social, prosperidad y una vida mejor como lema de campaña para el gobierno del momento. A partir de los objetivos de la estrategia se busca reducir la pobreza extrema, creación de nuevos empleos e incorporación de beneficios a familias (República Dominicana, 2015).

Como se evidencia, los programas de transferencia monetaria condicionada se han creado como respuesta a condiciones de pobreza en Latinoamérica y han demostrado una disminución importante de sus índices en la región, ello surge como una noción positiva en respuesta a fallas en los servicios de educación y salud que brinda el estado (Dapuez, 2016), pero se contribuye al mejoramiento de la calidad de vida de las familias en situación de pobreza.

Existen problemas que actualmente acaparan la atención de la sociedad, con consecuencias negativas en las poblaciones objeto de políticas y programas de desarrollo rural, los cuales van desde la corrupción, carencia de una estrategia clara de desarrollo rural, focalización y aplicación a grupos inespecíficos, falta de exigibilidad en los plazos 
de implementación y sus resultados concretos, dificultad de acceso y explotación adecuada de la tierra por parte de los campesinos, lo que se llamaría en este contexto el desarrollo económico real para los campesinos, poca participación de la sociedad civil, baja aplicabilidad de los programas de economía rural en contraste con la economía global, lo que conlleva a que sean inviables y la brecha cada vez mayor aunada a la pobreza rural que genera que los presupuestos destinados hacia proyectos de desarrollo rural se desvíen hacia proyectos sociales de corto plazo (Bandeira, Atance y Sumpsi, 2004; Rossel, 2012).

\section{Discusión}

Hablar ampliamente de políticas y programas gubernamentales para la salud rural sería posible si identificamos el enfoque que se ha estructurado de acuerdo con las intervenciones estatales que dan respuestas económicas y se logra la transformación en estrategias focalizadas hacia la salud rural. Si bien las ayudas monetarias para las regiones rurales representan un recurso valioso, se impactarían mejor los indicadores en salud ante programas de índole social, comunitario, participativo, ampliando el capital humano (Satterthwaite, 2004), lamentablemente para el sector rural la evidencia es reducida, lo que se considera prioritario teniendo en cuenta que Latinoamérica es un continente predominantemente rural.

Como medida para repensar la salud, de acuerdo con la evidencia latinoamericana en la creación y aplicación de políticas y programas específicos para la población campesina se debe diseñar e implementar un programa pensado en la noción moderna de desarrollo rural y con ello trabajar en la búsqueda de objetivos centrados en la población, por ello es indispensable introducir elementos culturales y hacerlas viables dentro de la política de estado. Necesariamente al hablar de una estrategia de aplicación rural, se debe tener conocimiento de la actividad económica predominante en la región para la generación de capacidad productiva, y así comprender culturalmente sus potencialidades y falencias para su desarrollo.

Con las reflexiones que se pueden desencadenar de la evidencia, es necesario trabajar por la viabilidad económica de políticas y programas para la reorganización en salud de la población campesina por medio del incentivo productivo, generando propuestas autosustentables. Se debe realizar un conocimiento del campo de acción, diálogos con líderes sociales de la región, entendiendo las necesidades identificadas y promoviendo la participación social (Ramírez, 2014); adicionalmente debe asumirse como un proyecto político para que adquiera peso y voz de manera bilateral, sin olvidar el respeto a los derechos fundamentales. 
Revista CIFE / ISSN: 0124-3551 e-ISSN: 2248-4914 / Bogotá-Colombia / Vol. 20 N.o 33 / julio-diciembre 2018 / pp. $79-94$

\section{Referencias bibliográficas}

Acosta, A. (2013). Colombia: escenario de las desigualdades. Revista de la Facultad de Ciencias Económicas y Administrativas, 14(1), 27. Recuperado de http://revistas.udenar. edu.co/index.php/rtend/article/view/974/1210

Acosta, M., Sáenz, M. D., Gutiérrez, B. y Bermúdez, J. L. (201 1). Sistema de salud de El Salvador. Salud Publica Mex, 53(2), 188-196. Recuperado de http://www.scielo.org. $\mathrm{mx} / \mathrm{pdf} / \mathrm{spm} / \mathrm{v} 53 \mathrm{~s} 2 / 14 . \mathrm{pdf}$

Arriagada, I., Aranda, F. y Divisi, M. (2005). Políticas y programas de salud en América Latina. Problemas y propuestas. CEPAL, Serie Políticas Sociales, 114. Recuperado de https://repositorio.cepal.org/bitstream/handle/11362/6119/1/S051049 es.pdf

Bandeira, P., Atance, I. y Sumpsi, J. M. (2004). Las políticas de desarrollo rural en América Latina: requerimientos de un nuevo enfoque. Cuaderno de Desarrollo rural, (51), 115-136.

BBC News Mundo. (2017). ¿Cuáles son los países de América Latina que más y menos invierten en salud y con qué resultados? Recuperado de https://www.bbc.com/mundo/ noticias-41399983

Breu, F., Guggenbichler, S. y Wollmann, J. (2003). La pobreza rural en América Latina: lecciones para una reorientación de las políticas. Encuentro de altos directivos sobre la mitigación de la pobreza rural. Recuperado de https://repositorio.cepal.org/bitstream/ handle/11362/6540/1/S037467 es.pdf

Brumer, A. (2003). Os rumos do mundo rural na América Latina no início do século XXI, num cenário de grandes transformações sociais, econômicas e políticas. Sociologias, (10), 14-25. Doi: https://doi.org/10.1590/S1517-45222003000200002

Canel, M.J. (2006). Comunicación política, una guía para su estudio y práctica. (Segunda edición). Madrid: Editorial Tecnos 2006. Biblioteca Universitaria. ISBN 9788430944408.

CEPAL. (2005). Tekopora 2005, programas de transferencias condicionadas, base de datos de programas de protección social no contributiva en América Latina y el Caribe. Paraguay. Recuperado de https://dds.cepal.org/bpsnc/programa?id=27

Comité Técnico Interministerial. (2014). Política Nacional de Desarrollo Rural. Chile. Recuperado de http://extwprlegs 1.fao.org/docs/pdf/chi142198.pdf 
Dapuez, A. (2016). Políticas de transferencias monetarias. Exportando expectativas en desarrollo. RUNA, archivo para las ciencias del hombre, 37(1), 53-69. Recuperado de http://revistascientificas.filo.uba.ar/index.php/runa/article/view/1503/2267

Decreto 2145. (2017). Por el cual se adopta el plan de revisión, evaluación y seguimiento de los programas y leyes que favorecen a las mujeres rurales y se crea el Comité Interinstitucional de seguimiento al Plan. Diario Oficial n. ${ }^{\circ}$ 50.453. Recuperado de http://www.funcionpublica.gov. co/eva/gestornormativo/norma.php?i=85748

Cecchini, S. y Madariaga, A. (2011). Programas de Transferencias Condicionadas, balance de la experiencia reciente en América Latina y el Caribe. Recuperado de https:/ / repositorio.cepal. org/bitstream/handle/11362/27854/S2011032 es.pdf

Felitti, K. (2009). Derechos reproductivos y políticas demográficas en América Latina. Revista de Ciencias Sociales, 35, 55-66. Doi: https://doi.org/13901249

Fondo de Cooperación para el Desarrollo Social. (2017). FONCODES. Ministerio de Desarrollo e Inclusión Social. Perú. Recuperado de http://www.foncodes.gob.pe/ portal/index.php/comunicacion-e-imagen/publicaciones?download=14470:memo ria-institucional-2016.

Gómez, J. (2016). Organización y descripción documental del Programa de Adquisición de Tierras. Revista Interamericana de Bibliotecología, 2, 16. Antioquia: INCORA. Doi: https://doi.org/10.17533/udea.rib.v39n2a08

Hernández, M. y Escobar, A. (2004). Resultados de la Evaluación Externa del Programa de Desarrollo Humano Oportunidades. Recuperado de https://publications.iadb.org/publications/spanish/document/Resultados-de-la-evaluaci\%C3\%B3n-externa-delprograma-de-desarrollo-humano-Oportunidades.pdf

Jaramillo, C. (2005). Red de Solidaridad Social en Colombia. Revista EAFIT, (105), 1-14.

Julca, M., Pardo, A. y Escalante, M. (2005). Programa nacional de manejo de cuencas hidrográficas y conservación de suelos PRONAMACHCS. Cajamarca. Recuperado de https://www. agrorural.gob.pe/dmdocuments/transparencia/memorias/Memoria 2005.pdf

Kliksberg, B. (2002). La inequidad en el campo de la salud pública en América Latina: una cuestión crucial. Economía y Sociedad, 7(19), 93-108. Recuperado de https://doaj. org/article/30abef38df87475cb98f09d3017bc14c

Ley 113. (2007). Por medio de la cual se crea e implementa el programa Agro, Ingreso Seguro - AIS. Diario Oficial n. ${ }^{\circ}$ 46.595. Congreso de la República. Recuperado de https://www. ica.gov.co/getattachment/235a5c55-4109-4612-9145-ff54fa9bfe5d/2007L1133.aspx 
Revista CIFE / ISSN: 0124-3551 e-ISSN: 2248-4914 / Bogotá-Colombia / Vol. 20 N.o 33 / julio-diciembre 2018 / pp. $79-94$

Machado, A. (2010). Lecciones sobre desarrollo rural, retos y enfoques. Lecciones del desarrollo rural, desafíos y avances. Agronomía Colombiana, 28(3), 428-434. Recuperado de http://www.scielo.org.co/scielo.php? script=sci arttextypid=S0120$\underline{99652010000300010 y \operatorname{lng}=\text { enytlng }=\text { en }}$

Martínez, D., Borja, T., Medellín, N. y Cueva, P. (2017). Cómo funciona el bono de desarrollo humano - Mejores prácticas en la implementación de programas de transferencias monetarias condicionadas en América Latina y el Caribe. Banco Interamericano de Desarrollo. Recuperado de https://publications.iadb.org/publications/spanish/ document $/ \% \mathrm{C} 2 \% \mathrm{BFC} \% \mathrm{C} 3 \% \mathrm{~B} 3 \mathrm{mo} \%$-funciona-el-Bono-de-Desarrollo-Humano-Mejores-pr $\% \mathrm{C} 3 \% \mathrm{~A} 1$ cticas-en-la-implementaci $\% \mathrm{C} 3 \% \mathrm{~B} 3 \mathrm{n}$-de-Programas-de-Transfe$\underline{\text { rencias-Monetarias-Condicionadas-en-Am } \% \mathrm{C} 3 \% \text { A9rica-Latina-y-el-Caribe.pdf }}$

Medellín, N. y Sánchez, F. (2015). Cómo funciona más familias en acción - Mejores prácticas en la implementación de programas de transferencias monetarias condicionadas en América Latina y el Caribe. Banco Interamericano de Desarrollo. Recuperado de https:// publications.iadb.org/publications/spanish/document $/ \% \mathrm{C} 2 \% \mathrm{BFC} \% \mathrm{C} 3 \% \mathrm{~B} 3 \mathrm{mo}-$ funciona-M $\% \mathrm{C} 3 \% \mathrm{~A} 1 \mathrm{~s}-\mathrm{Familias}-\mathrm{en}-\mathrm{Acci} \% \mathrm{C} 3 \% \mathrm{~B} 3 \mathrm{n}-\mathrm{Mejores}-\mathrm{pr} \% \mathrm{C} 3 \% \mathrm{~A} 1 \mathrm{cticas}-$ en-la-implementaci $\% \mathrm{C} 3 \% \mathrm{~B} 3 \mathrm{n}$-de-programas-de-transferencias-monetarias-condicionadas-en-Am\%C3\%A9rica-Latina-y-el-Caribe.pdf

Ministerio de Agricultura. (s. f.). Lineamientos estratégicos 2014 - 2018. Instituto de Desarrollo Agropecuario INDAP. Recuperado de https://www.indap.gob.cl/docs/ default-source/Lineamientos-Estrat $\% \mathrm{C} 3 \%$ A9gicos/lineamientos-estrategicos2014-2018-vf.pdf?sfvrsn=0

Ministerio de Agricultura. (2018). Política pública en pro de la mujer rural. Recuperado de https://www.minagricultura.gov.co/noticias/Paginas/Política-pública-en-pro-dela-mujer-rural.aspx

Ministerio de Medio Ambiente. (2011). Bolsa Verde. Recuperado de http://www.mma. gov.br/desenvolvimento-rural/bolsa-verde

Organización Mundial de la Salud. (2003). Influencia de la pobreza en la salud. Recuperado de http://apps.who.int/gb/archive/pdf files/EB113/seb11312.pdf

Organización Panamericana de la Salud. (2008). Perfil de los sistemas de salud de Nicaragua. Monitoreo y análisis de los procesos de cambio y reforma. (Tercera edición). Nicaragua. Recuperado de https://www.paho.org/nic/index.php?option=com

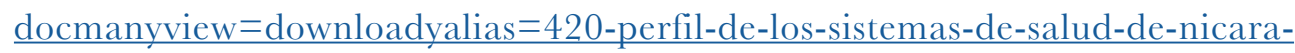
guaycategory slug=sistemas-de-servicios-de-salud-y-tecnologiayItemid=235 
Ortiz, L., Pérez, D. y Tamez, S. (2014). Desigualdad socioeconómica y salud en México. Rev Med Inst Mex Seguro Soc, 3(53), 336-347. Recuperado de http://web.a.ebscohost. com.ezproxy.unal.edu.co/ehost/pdfviewer/pdfviewer?vid=1ysid=d8fe49cc-84bd4c4e-a508-2a337f7749be\%40sdc-v-sessmgr06

Pautassi, L., Arcidiácono, P. y Straschnoy, M. (2013). Asignación Universal por Hijo para la Protección Social de la Argentina. Santiago de Chile: CEPAL, Unicef. Recuperado de https://repositorio.cepal.org/bitstream/handle/11362/6193/1/LCL3662_es.pdf

Pérez, S. L. y Arrivillaga, M. (2017). Redes integradas de servicios de salud en el marco de la atención primaria en salud en países seleccionados de América Latina. Salutem Scientia Spiritus, 3(2), 32-49. Recuperado de http://revistas.javerianacali.edu.co/index.php/salutemscientiaspiritus/article/view/1791/pdf 1

Ramírez, C. (2014). Critical reflections on the New Rurality and the rural territorial development approaches in Latin America. Agronomía Colombiana, 32(1), 122-129. Doi: https://doi.org/10.15446/agron.colomb.v32n1.41218

República Dominicana. (2015). Progresando con solidaridad. Recuperado de https://progresandoconsolidaridad.gob.do/nosotros/quienes-somos/

República de Nicaragua. (1998). Creación del Fondo Social Suplementario. Recuperado de http://legislacion.asamblea.gob.ni/normaweb.nsf/(\$All)/A522EE8A2354B6DF06 2570A10057A32E? OpenDocument

República de Panamá. (2009). Red de oportunidades empresariales para familias pobres. (Documento oficial). España. Recuperado de http://www.mdgfund.org/sites/default/files/Signed JP Panama DevandPS.pdf

Reyes, G. (2014). América Latina 2010: inversión social como porcentaje del PIB. Revista de Ciencias Sociales, 20(2), 279-287. Doi: https://doi.org/28031815007

Rodríguez, M. A. (2011). Democracia en América Latina, apertura económica, populismo y crisis. Revista de Ciencias Económicas, 29(2), 27-50. Recuperado de https://revistas. ucr.ac.cr/index.php/economicas/article/view/7015

Rodríguez, D. y Benavidez, J. (2016). Salud y ruralidad en Colombia: análisis desde los determinantes sociales de la salud. Facultad Nacional de Salud Pública, 34(3), 359371. Recuperado de http://www.scielo.org.co/pdf/rfnsp/v34n3/0120-386Xrfnsp-34-03-00359.pdf

Romero, J., Ferrero, G., Pérez, F., Baselga, P. y Rodríguez, T. (2000). La iniciativa por el desarrollo rural de Nicaragua: un proceso de concertación para implementación de políticas de 
Revista CIFE / ISSN: 0124-3551 e-ISSN: 2248-4914 / Bogotá-Colombia / Vol. 20 N.o 33 / julio-diciembre 2018 / pp. $79-94$ desarrollo rural en Nicaragua. Nicaragua. Recuperado de http://www.fundacionetea.org/ media/File/Microsoft Word - COmunicacion CIER JRomero GFerrero corregido.doc.pdf

Rossel, G. (2012). Protección social y pobreza rural en América Latina. CEPAL.

Salgado, N., González, T., Jaurégui, B. y Bonilla, P. (2005). No hacen viejos los años, sino los daños, envejecimiento y salud en varones rurales. Salud Pública de México, 47(4), 294-302. Recuperado de http://www.scielo.org.mx/scielo.php?script=sci arttextypi $\underline{\mathrm{d}=\mathrm{S} 0036-36342005000400007}$

Salvador, G. (2012). Comunidades solidarias. El Salvador: Secretaria Técnica de la Presidencia del Salvador.

Sánchez, A., Meléndez, G. y Behrman, J. (2016). The Impact of the Funtos Conditional Cash Transfer Programme in Peru on Nutritional and Cognitive Outcomes: Does the Age of Exposure Matter. London. Recuperado de http://younglives.org.uk/sites/www.younglives. org.uk/files/YL-WP153-Impact $\% 20$ of $\% 20 J u n t o s \% 20$ on $\% 20$ nutrition $\% 20$ and $\% 20^{\circ}$ education $\% 20 \mathrm{in} \% 20$ Peru.pdf

Satterthwaite, D. (2004). Metas de desarrollo para el milenio (MDM) y reducción de la pobreza. Cuaderno Urbano, 4(4), 231-272. Recuperado de http://revistas.unne.edu. ar/index.php/crn/article/view/1051

Tokman, V. (2006). Inserción laboral, mercados de trabajo y protección social. Santiago de Chile. Recuperado de https://repositorio.cepal.org/bitstream/handle/11362/3676/1/ $\underline{\text { S2006023_es.pdf }}$

Valencia, E. (2008). Las transferencias monetarias condicionadas como política social en América Latina. Un balance: aportes, límites y debates. Annual Review of Sociology, 34(1), 499-524. DOI: https://doi.org/10.1146/annurev.soc.052708.074708

Zurbriggen, C. y Travieso, E. (2016). Hacia un nuevo Estado desarrollista: desafíos para América Latina. Perfiles Latinoamericanos, 24(47), 259-281. DOI: https://doi. org/10.18504/pl2447-004-2016 